\title{
S-Shaped Conformation of Quaterthiophene Molecular Backbone in Two- Dimensional BisTerpyridine-Derivative Self-Assembled Nanoarchitecture
}

Yann Kervella, ${ }^{\mathrm{a}}$ Ekaterina Shilova, ${ }^{\mathrm{b}}$ Sylvain Latil, ${ }^{\mathrm{c}}$ Bruno Jousselme ${ }^{\mathrm{a}, \mathrm{d}}$ and Fabien Silly ${ }^{\mathrm{e}}$

${ }^{a}$ CEA Grenoble INAC/SPrAM UMR 5819 CEA-CNRS-Univ. J. Fourier-Grenoble 1, LEMOH, 17 Rue des Martyrs, 38054 Grenoble Cedex 9, France.

${ }^{b}$ Ajelis, 86 rue de Paris, F-94100 Orsay, France

${ }^{c}$ MSIN, SPEC, CEA, CNRS, Université Paris-Saclay, CEA Saclay F-91191 Gif sur Yvette, France

${ }^{d}$ LICSEN, NIMBE, CEA, CNRS, Université Paris-Saclay, CEA Saclay F-91191 Gif sur Yvette, France.

${ }^{e}$ TITANS, SPEC, CEA, CNRS, Université Paris-Saclay, CEA Saclay F-91191 Gif sur Yvette, France. Fax: +33 16908 8446; Tel: +33 16908 8019; E-mail: fabien.silly@cea.fr

\section{SUPPORTING INFORMATION}

\section{Synthesis:}

The targeted compound 4'-(3',4'-Dihexyloxy-5,2':5',2"'5,',2,"'-quaterthien-2,5'”'diyl)-bis(2,2':6',2''-terpyridine) (4T-bisTerpy) was synthesized according scheme S1. The 4'-(5-Bromo-2-thienyl)-2,2':6',2'-terpyridine (2) was synthesized according to the Kröhnke's method as already described before. ${ }^{1}$ Compare to the previous description, methanol was used as solvent for the two steps reactions to obtain directly by precipitation the chalcone 1 and to increase the global yield of compound 2. In the second time, 3-bromothiophene was converted to 3-hexyloxythiophene (3) in the presence of sodium hexanoate and Cul. Compound 3 was treated first with LDA, then coupled with copper iodide to give 2,2'-bi(4-hexyloxythiophene) (4). Dilithiation of the bi-thiophene derivative and reaction with tributyltin chloride afforded the bis(tributylstannyl) derivative 5. A Stille coupling between 2 and $\mathbf{5}$ in the presence of palladium catalyst lead to 4T-bisTerpy. 


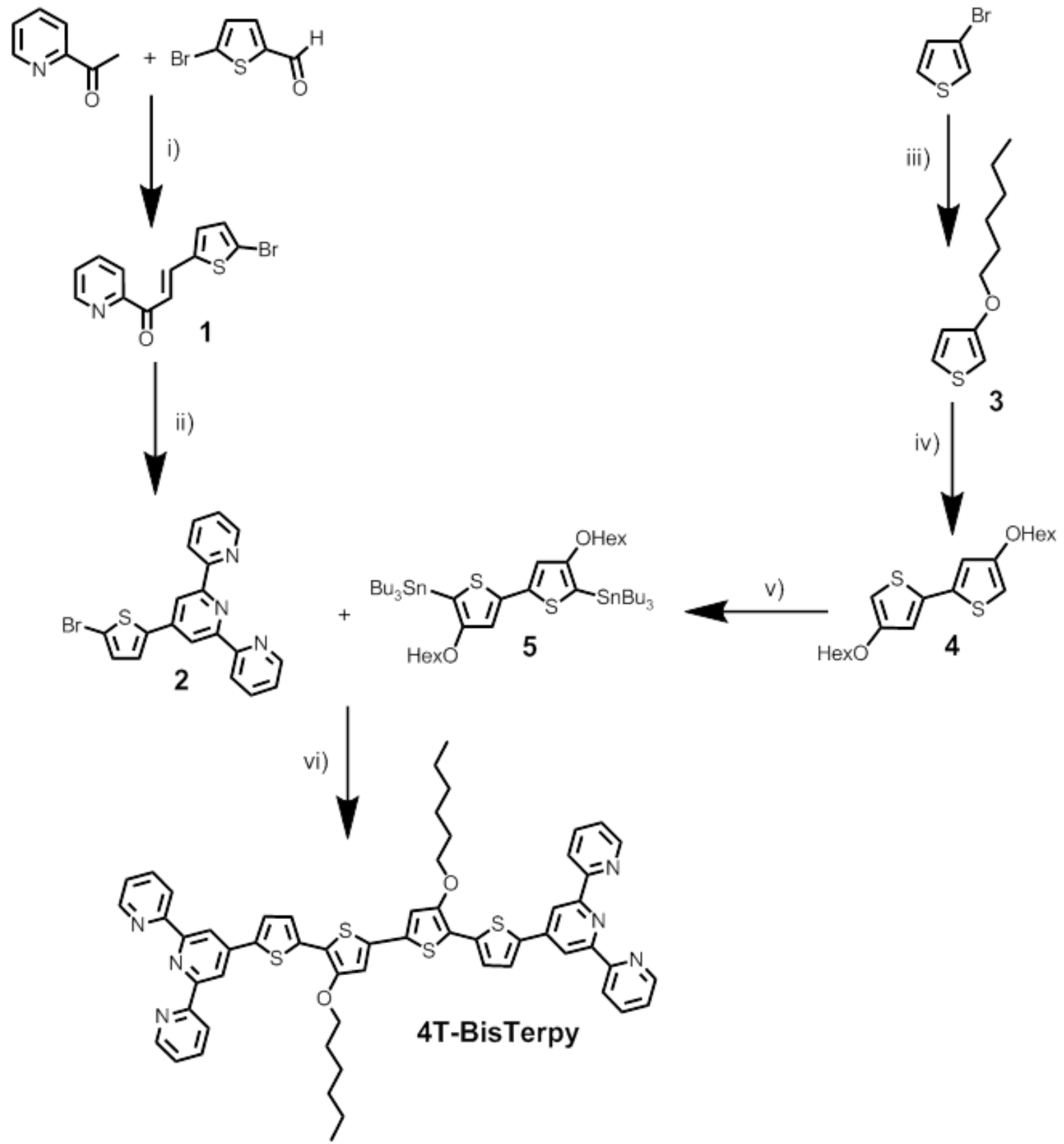

Scheme S1. Synthesis of 4T-bisTerpy. i) $\mathrm{KOH}, \mathrm{MeOH} / \mathrm{H}_{2} \mathrm{O}, 0{ }^{\circ} \mathrm{C}$ (80\%). ii) $\mathrm{PPI}$, $\mathrm{NH}_{4} \mathrm{OAc}, \mathrm{MeOH}, \operatorname{reflux}(55 \%)$. iii) 1. $\mathrm{Na}, 1-\mathrm{Hexanol}, 90^{\circ} \mathrm{C}$; 2. Cul, DMF, $90^{\circ} \mathrm{C}, \mathrm{N}_{2}$ (55\%). iv) 1. $n$-BuLi, THF, $-78^{\circ} \mathrm{C}$; 2. $\mathrm{CuCl}_{2}, \mathrm{~N}_{2}$ (47\%). v) 1. $n$-BuLi, THF, $-78^{\circ} \mathrm{C} ; 2$. $\mathrm{Bu}_{3} \mathrm{SnCl}, \mathrm{N}_{2}$. vi) 4, [Pd(PPh$\left.)_{4}\right]$, DMF, $\operatorname{Ar}(35 \%)$. 


\section{Experimental Section}

\section{Characterization techniques:}

NMR spectra were recorded with a Bruker AVANCE AC $200 \mathrm{MHz}\left({ }^{1} \mathrm{H}, 200.13 \mathrm{MHz}\right.$ and ${ }^{13} \mathrm{C}, 50.32 \mathrm{MHz}$ ). Chemical shifts are given in ppm relative to TMS. UV-Vis spectra were recorded with a Perkin Elmer paragon 500 spectrometer with Bio-Tek UVIKON XS spectrometer. Electrospray mass spectra (MS-ESI) were acquired in the positive mode on a LCQ-ion trap Thermofinnigan spectrometer equipped with an electrospray source and fast atom bombardment mass spectra (MS-FAB) were recorded with a AIE Kratos MS 50 mass spectrometer fitted with an lon Tech Ltd gun. A standard FAB source was used and m-nitrobenzylalcohol (NBA) was the liquid matrix. Elemental analyses were carried out by the Analytical Service of CNRS Vernaison (France).

\section{Reagents and chemicals}

All reagents and chemicals were purchased from Aldrich. Reagents and chemicals were used as received until it was mentionned. Pyridacyl pyridinium iodide (PPI) ${ }^{2}$ was prepared according to known procedure.

(E)-3-(5-Bromo-2-thienyl)-1-(2-pyridyl)prop-2-en-1-one (1). $\mathrm{KOH}$ (2.94 g, 52.3 $\mathrm{mmol})$ was dissolved in a mixture of methanol and water $(5: 1,120 \mathrm{~mL})$ and cooled to $0{ }^{\circ} \mathrm{C}$. Afterward, 3-bromothiophene-2-carboxaldehyde $(10 \mathrm{~g}, 52.3 \mathrm{mmol})$ and 2acetylpyridyne $(6.34 \mathrm{~g}, 52.3 \mathrm{mmol})$ were charged successively and the mixture was stirred $3 \mathrm{H}$ at $0{ }^{\circ} \mathrm{C}$. The formed precipitate was filtered and washed with cold methanol to get 1 as yellow-green solid $(15.4 \mathrm{~g}, 80 \%)$. ${ }^{1} \mathrm{H}$ NMR $\left(\mathrm{CDCl}_{3}\right): \delta=8.72$ (ddd, $2 \mathrm{H},{ }^{3} \mathrm{~J}=4.8 \mathrm{~Hz},{ }^{4} \mathrm{~J}=1.8 \mathrm{~Hz},{ }^{5} \mathrm{~J}=0.9 \mathrm{~Hz}$ ), 8.15 (ddd, $2 \mathrm{H},{ }^{3} \mathrm{~J}=7.6 \mathrm{~Hz},{ }^{4} \mathrm{~J}=1.2$ $\left.\mathrm{Hz},{ }^{5} \mathrm{~J}=0.9 \mathrm{~Hz}\right), 7.98\left(\mathrm{~d}, 1 \mathrm{H},{ }^{3} \mathrm{~J}=15.8 \mathrm{~Hz}\right), 7.88\left(\mathrm{~d}, 1 \mathrm{H},{ }^{3} \mathrm{~J}=15.8 \mathrm{~Hz}\right), 7.86(\mathrm{td}, 2 \mathrm{H}$, ${ }^{3} \mathrm{~J}=7.6 \mathrm{~Hz},{ }^{4} \mathrm{~J}=1.8 \mathrm{~Hz}$ ), 7.48 (ddd, $2 \mathrm{H},{ }^{3} \mathrm{~J}=7.6 \mathrm{~Hz},{ }^{3} \mathrm{~J}=4.8 \mathrm{~Hz},{ }^{4} \mathrm{~J}=1.2 \mathrm{~Hz}$ ), 7.13 $\left(\mathrm{d}, 1 \mathrm{H},{ }^{3} \mathrm{~J}=3.9 \mathrm{~Hz}\right), 7.04\left(\mathrm{~d}, 1 \mathrm{H},{ }^{3} \mathrm{~J}=3.9 \mathrm{~Hz}\right)$.

4'-(5-Bromo-2-thienyl)-2,2':6',2'-terpyridine (2). 1 (2.94 g, $10 \mathrm{mmol}$ ), PPI (3.26 g, $10 \mathrm{mmol}$ ) and $\mathrm{NH}_{4} \mathrm{OAc}(771 \mathrm{mg}, 10 \mathrm{mmol})$ were refluxed in methanol under Argon. After $6 \mathrm{H}$, the precipitate obtained was filtered and the methanol solution was refluxed again. After $6 \mathrm{H}$, the new precipitate was filtered again. This was repeated 
until no more precipitate appeared. The grey precipitates were gathered together and washed with cold methanol and filtered on alumina plug (eluent: $\mathrm{CHCl}_{3}$ ) to give 2 as a white solid (2.1 g, 53 \%). m.p. $=224-226{ }^{\circ} \mathrm{C} .{ }^{1} \mathrm{H} \mathrm{NMR}\left(\mathrm{CDCl}_{3}\right): \delta=8.72\left(\mathrm{ddd}, 2 \mathrm{H},{ }^{3} \mathrm{~J}\right.$ $=4.8 \mathrm{~Hz},{ }^{4} \mathrm{~J}=1.8 \mathrm{~Hz},{ }^{5} \mathrm{~J}=0.9 \mathrm{~Hz}$ ), 8.62 (ddd, $2 \mathrm{H},{ }^{3} \mathrm{~J}=7.5 \mathrm{~Hz},{ }^{4} \mathrm{~J}=1.0 \mathrm{~Hz},{ }^{5} \mathrm{~J}=0.9$ $\mathrm{Hz}), 8.59$ (s, 2H), $7.87\left(\mathrm{td}, 2 \mathrm{H},{ }^{3} \mathrm{~J}=7.5 \mathrm{~Hz},{ }^{4} \mathrm{~J}=1.8 \mathrm{~Hz}\right), 7.51\left(\mathrm{~d}, 1 \mathrm{H},{ }^{3} \mathrm{~J}=4.0 \mathrm{~Hz}\right)$, 7.35 (ddd, $\left.2 \mathrm{H},{ }^{3} \mathrm{~J}=7.5 \mathrm{~Hz},{ }^{3} \mathrm{~J}=4.8 \mathrm{~Hz},{ }^{4} \mathrm{~J}=1.2 \mathrm{~Hz}\right), 7.11\left(\mathrm{~d}, 1 \mathrm{H},{ }^{3} \mathrm{~J}=4.0 \mathrm{~Hz}\right)$. UV/Vis $\left(\mathrm{CH}_{2} \mathrm{Cl}_{2}\right): \lambda=288 \mathrm{~nm}(\log \varepsilon=4.59) ; 311$ (sh).

3-Hexyloxythiophene (3). In a three neck under argon atmosphere, sodium (1.88 g, $82 \mathrm{mmol}$ ) was charged to 1 -Hexanol $(35 \mathrm{~mL})$. This mixture was heated at $90^{\circ} \mathrm{C}$ until no metal sodium left. Then, hexanol excess was removed with vacuum distillation to get the sodium hexanoate. 3-Bromothiophene (12 g, $73.6 \mathrm{mmol})$ and Cul (2.6 g, 13.6 mmol) was added to the white solid obtained previously dissolved in dry DMF (40 $\mathrm{mL}$ ). The mixture was heated overnight at $90^{\circ} \mathrm{C}$ under argon. Afterward, water (100 $\mathrm{mL}$ ) was added and the aquous phase was extracted with Hexane. The organic phases were gathered together, washed with water, dried $\left(\mathrm{Na}_{2} \mathrm{SO}_{4}\right)$ and concentrated. The purification was realized by vacuum distillation $(0.1 \mathrm{~mm}$ of $\mathrm{Hg})$, the first fraction was the excess of hexanol and the second fraction gave $3\left(80^{\circ} \mathrm{C}\right.$ at 1 $\mathrm{mm}$ of $\mathrm{Hg})$ as colorless oil $(7.5 \mathrm{~g}, 55 \%)$. ${ }^{1} \mathrm{H}$ NMR $\left(\mathrm{CDCl}_{3}\right): \delta=7.16\left(\mathrm{dd}, 1 \mathrm{H},{ }^{3} \mathrm{~J}=5.2\right.$ $\left.\mathrm{Hz},{ }^{4} \mathrm{~J}=3.0 \mathrm{~Hz}\right), 6.75\left(\mathrm{dd}, 1 \mathrm{H},{ }^{3} \mathrm{~J}=5.2 \mathrm{~Hz},{ }^{4} \mathrm{~J}=1.5 \mathrm{~Hz}\right), 6.22\left(\mathrm{dd}, 1 \mathrm{H},{ }^{4} \mathrm{~J}=3.0 \mathrm{~Hz},{ }^{4} \mathrm{~J}\right.$ $=1.5 \mathrm{~Hz}), 3.94\left(\mathrm{t}, 2 \mathrm{H},{ }^{3} \mathrm{~J}=6.4 \mathrm{~Hz}\right), 1.77\left(\mathrm{qu}, 2 \mathrm{H},{ }^{3} \mathrm{~J}=6.4 \mathrm{~Hz}\right), 1.50-1.20(\mathrm{~m}, 6 \mathrm{H})$, $0.90\left(\mathrm{t}, 3 \mathrm{H},{ }^{3} \mathrm{~J}=6.2 \mathrm{~Hz}\right)$.

2,2'-Bi(4-hexyloxythiophene) (4). A LDA solution prepared with n-Butyllithium (3.2 $\mathrm{mL}, 2.5 \mathrm{M}$ in hexane, $8 \mathrm{mmol}$ ) added dropwise to a stirred solution of diisopropylamine $(1.15 \mathrm{~mL}, 8.14 \mathrm{mmol})$ in dry THF $(5 \mathrm{~mL})$ under argon at $0^{\circ} \mathrm{C}$ was cannulated to a solution of $3(1.5 \mathrm{~g}, 8.14 \mathrm{mmol})$ in dry THF $(20 \mathrm{~mL})$ at $-78^{\circ} \mathrm{C}$ under inert atmosphere. This mixture was stirred $1 \mathrm{H}$ at this tempetraure then $\mathrm{CuCl}_{2}$ solid was added and the solution warmed to room temperature. After $12 \mathrm{H}$ of stirring, water $(50 \mathrm{~mL})$ and diethyl ether was added. The organic phase was separated by decantation and the aqueous phase was extracted two more times with diethyl ether. The organics phases were collected, sewpt through celite, dried $\left(\mathrm{Na}_{2} \mathrm{SO}_{4}\right)$ and concentrated. Chromatography on silica gel (1:9 $\mathrm{CH}_{2} \mathrm{Cl}_{2} /$ petroleum ether) gave 4 as a yellow solid (650 mg, 47\%). ${ }^{1} \mathrm{H}$ NMR $\left(\mathrm{CDCl}_{3}\right): \delta=6.81\left(\mathrm{~d}, 2 \mathrm{H},{ }^{4} \mathrm{~J}=1.8 \mathrm{~Hz}\right), 6.10(\mathrm{~d}$, $\left.2 \mathrm{H},{ }^{4} \mathrm{~J}=1.8 \mathrm{~Hz}\right), 3.92\left(\mathrm{t}, 4 \mathrm{H},{ }^{3} \mathrm{~J}=6.5 \mathrm{~Hz}\right), 1.76\left(\mathrm{qu}, 4 \mathrm{H},{ }^{3} \mathrm{~J}=6.3 \mathrm{~Hz}\right), 1.50-1.20(\mathrm{~m}$, $12 \mathrm{H}), 0.91\left(\mathrm{t}, 6 \mathrm{H},{ }^{3} \mathrm{~J}=6.4 \mathrm{~Hz}\right)$. 


\section{4'-(3',4"'-Dihexyloxy-5,2':5',2':5', 2,'-quaterthien-2,5'"-diyl)-bis(2,2':6',2"'-}

terpyridine) (4T-bisTerpy). A solution of n-BuLi $2.5 \mathrm{M}$ in hexane ( $1 \mathrm{~mL}, 2.2$ eq.) was added dropwise to a solution of $4(500 \mathrm{mg}, 1.36 \mathrm{mmol})$ in anhydrous THF $(10 \mathrm{~mL})$ under $\mathrm{Ar}$ at room temperature. After $30 \mathrm{~min}$ of stirring, $\mathrm{Bu}_{3} \mathrm{SnCl}(0.9 \mathrm{~mL}, 2.4$ eq.) was slowly added and the reaction mixture was stirred for $1 \mathrm{~h}$ at $30^{\circ} \mathrm{C}$. After dilution with $\mathrm{CH}_{2} \mathrm{Cl}_{2}$ (60 mL), the organic phase was successively washed with a satured aqueous solution of $\mathrm{NH}_{4} \mathrm{Cl}$ and water, dried over $\mathrm{Na}_{2} \mathrm{SO}_{4}$ and evaporated in vacuo. The 5,5'Bis(tributylstannyl)-2,2'-bi(4-hexyloxythiophene) (5) obtained was directly used in the following step without further purification. A mixture of $5(1.1 \mathrm{~g}, 1.16 \mathrm{mmol}), 2(1 \mathrm{~g}$, 2.2 eq.) and $\mathrm{Pd}\left(\mathrm{PPh}_{3}\right)_{4}(156 \mathrm{mg}, 0.1$ eq.) in anhydrous $\mathrm{DMF}(10 \mathrm{~mL})$ was heated overnight at $100^{\circ} \mathrm{C}$ under a $\mathrm{Ar}$ atmosphere. Then, water was added to the mixture to get brown solid which was filtered, washed with water, methanol and diethyl ether. Afterward the solid was dissolved in hot $\mathrm{CHCl}_{3}$ and filtered on alumina plug $\left(\mathrm{CHCl}_{3}\right.$ then $\left.\mathrm{CHCl}_{3} / \mathrm{THF}(9: 1)\right)$ to get 4T-bisTerpy as purple solid (400 mg, 35\%). m.p. > $250^{\circ} \mathrm{C},{ }^{1} \mathrm{H}$ NMR $\left(\mathrm{CDCl}_{2}-\mathrm{CDCl}_{2}\right): \delta=8.72\left(\mathrm{~d}, 4 \mathrm{H},{ }^{3} \mathrm{~J}=3.8 \mathrm{~Hz}\right), 8.63(\mathrm{~s}, 4 \mathrm{H}), 8.60(\mathrm{~d}$, $4 \mathrm{H},{ }^{3} \mathrm{~J}=7.0 \mathrm{~Hz}$ ), $7.88\left(\mathrm{t}, 4 \mathrm{H},{ }^{3} \mathrm{~J}=7.0 \mathrm{~Hz}\right.$ ), $7.71\left(\mathrm{~d}, 2 \mathrm{H},{ }^{3} \mathrm{~J}=3.8 \mathrm{~Hz}\right), 7.36$ (dd, $4 \mathrm{H},{ }^{3} \mathrm{~J}=$ $\left.7.0 \mathrm{~Hz},{ }^{3} \mathrm{~J}=3.8 \mathrm{~Hz}\right), 7.28\left(\mathrm{~d}, 2 \mathrm{H},{ }^{3} \mathrm{~J}=3.8 \mathrm{~Hz}\right), 6.96(\mathrm{~s}, 2 \mathrm{H}), 4.21\left(\mathrm{t}, 4 \mathrm{H},{ }^{3} \mathrm{~J}=6.2 \mathrm{~Hz}\right)$, 1.92 (qu, $4 \mathrm{H},{ }^{3} \mathrm{~J}=6.4 \mathrm{~Hz}$ ), $1.75-1.27(\mathrm{~m}, 12 \mathrm{H}), 0.90\left(\mathrm{t}, 6 \mathrm{H},{ }^{3} \mathrm{~J}=6.6 \mathrm{~Hz}\right) .{ }^{13} \mathrm{C} \mathrm{NMR}$ $\left(\mathrm{CDCl}_{2}-\mathrm{CDCl}_{2}\right): \delta=157.5,155.1,150.8,144.8,140.6,138.5,136.7,134.5,127.9$, $125.3,125.2,122.9,118.1,115.9,114.7,73.9,33.2,31.2,27.4,24.2,15.8$. UV/Vis $\left(\mathrm{CH}_{2} \mathrm{Cl}_{2}\right): \lambda=479(\log \varepsilon=4.74), \lambda=278(\log \varepsilon=4.65)$. ESI-MS m/z : $993[\mathrm{M}+\mathrm{H}]^{+}$. Anal. for $\mathrm{C}_{58} \mathrm{H}_{52} \mathrm{O}_{2} \mathrm{~N}_{6} \mathrm{~S}_{4}$ (calcd) : C 70.11 (70.13) H 5.62 (5.28), N 7.96 (8.46), S 12.51 (12.91). 


\section{Calculations:}

In order to understand how the conformation of the quaterthiophene skeleton affects the energetics of the molecular adsorption we have firstly calculated the total energies of the optimized structure for all the I, C, S, L, D conformations ( $\left.E_{\text {gas }}\right)$. Secondly, we have optimized the molecules with different conformations, when adsorbed on a graphitic layer $\left(E_{\text {total }}\right)$, Figure $S 2$. By subtracting the total energy of the isolated graphitic layer supercell, the adsorption energy $\left(E_{a d h}\right)$ can be determined. Each structural optimisation was performed until forces get smaller than $10^{-4} \mathrm{eV} / \AA$. These simulations were carried out using the DFTB+ code, ${ }^{3}$ within its self-consistent charge implementation. ${ }^{4}$ The dispersion forces correction ${ }^{5}$ were added, ensuring correct predictions of the interaction between the molecule and the graphitic layer. Results are presented in the table below.
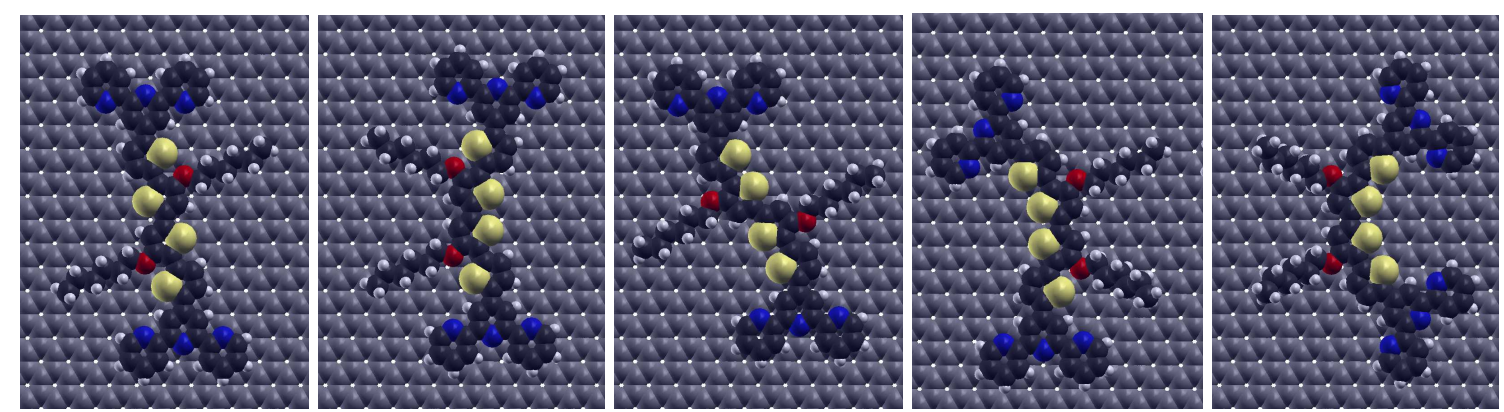

Figure S2 : Relaxed Molecule on graphitic sheet (I, C, S, L and D conformations)

\begin{tabular}{|c|c|c|c|c|}
\hline Scheme & Conformation & $\mathrm{E}_{\text {Gas }}(\mathrm{eV})$ & $\mathrm{E}_{\text {Total }}(\mathrm{eV})$ & $\mathrm{E}_{\text {Adh }}(\mathrm{eV})$ \\
\hline & $\mathrm{I}$ & -4081.153 & -22983.343 & -5.021 \\
\hline & $\mathrm{C}$ & -4081.123 & -22983.310 & -5.017 \\
\hline & $\mathrm{S}$ & -4081.105 & -22983.280 & -5.006 \\
\hline
\end{tabular}

Table I: Energy 


\section{STM characterization:}

Molecular self-assembly at the tetradecane-graphite and -Tetrahydronaphthalenegraphite interface:
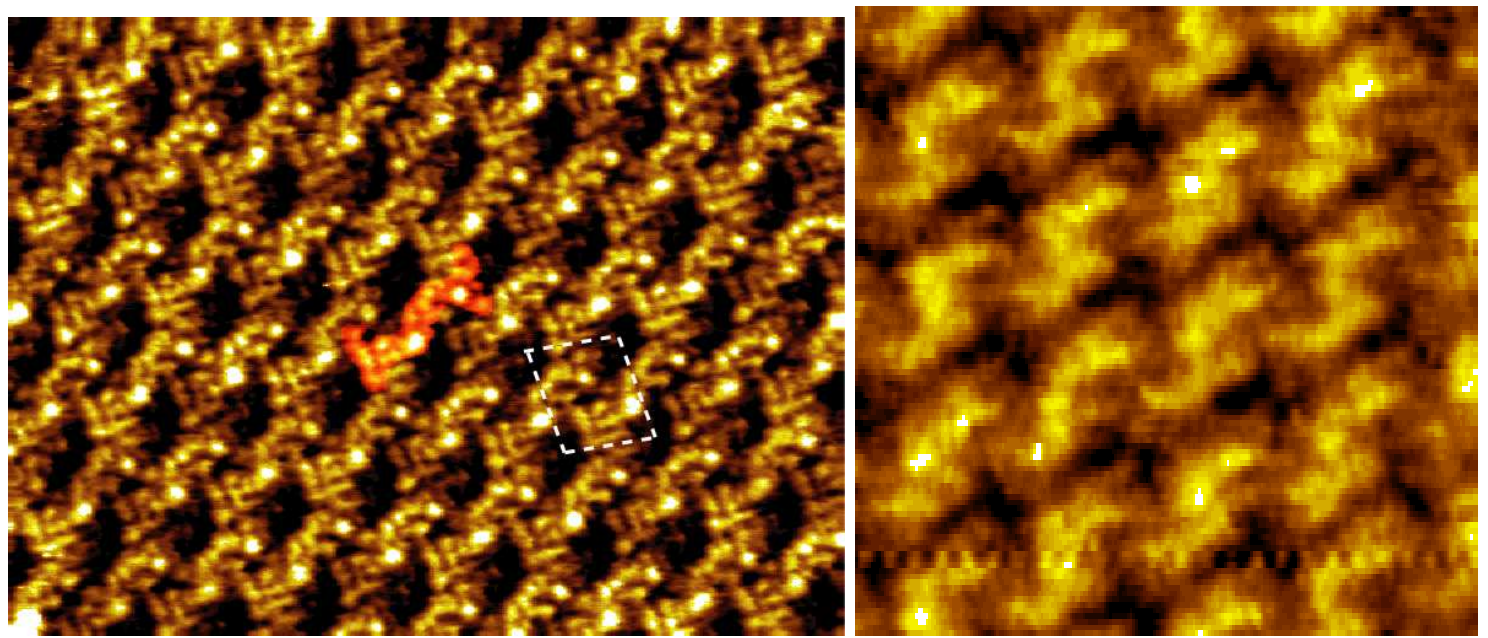

Figure S3: (a) STM image of molecular self-assembly at the tetradecane-graphite interface, $15 \times 10 \mathrm{~nm}^{2}, V_{s}=0.68 \mathrm{~V}, I_{t}=9 \mathrm{pA}$ (left) and at the -Tetrahydronaphthalene-graphite interface, $6 \times 6$ $\mathrm{nm}^{2}, V_{s}=0.70 \mathrm{~V}, \mathrm{I}_{t}=9 \mathrm{pA}$ (right). A single molecule is colored in red color in the organic layer. The unit cell is represented by dotted white lines.

The Fig.S3 shows that the molecules also self-assemble into a porous structure at the tetradecane-graphite and -Tetrahydronaphthalene-graphite interface. The molecules also adopt the S-conformation in this structure.

\section{REFERENCES:}

1 R. López, D. Villagra, G. Ferraudi, S. A. Moya, J. Guerrero, Inorg. Chim. Acta, 2004, 357, 3525.

2 J. Polin, E. Schmohel, V. Balzani, J. Chem. Soc., Dalton Trans., 2000, 445.

3 B. Aradi, B. Hourahine, and Th. Frauenheim. J. Phys. Chem. A, 2007, 111, 5678.

4 M. Elstner, D. Porezag, G. Jungnickel, J. Elsner, M. Haugk, T. Frauenheim, S. Suhai, G. Seifert, Phys. Rev. B, 1998, 58, 7260.

5 M. Elstner, P. Hobza, T. Frauenheim, S. Suhai, E. Kaxiras. J. Chem. Phys., 2001, $114,5149$. 